\title{
openheart A pilot study of angiotensin converting enzyme (ACE) genotype and return of spontaneous circulation following out-of-hospital cardiac arrest
}

\author{
Thomas W Lindner, ${ }^{1}$ Charles D Deakin, ${ }^{2,3,4}$ Hildegunn Aarsetøy, ${ }^{5}$ \\ Sten Rubertsson, ${ }^{6}$ Jon-Kenneth Heltne, ${ }^{7,8}$ Eldar Søreide ${ }^{1,8}$
}

To cite: Lindner TW, Deakin CD, Aarsetøy $\mathrm{H}$, et al. A pilot study of angiotensin converting enzyme (ACE) genotype and return of spontaneous circulation following out-of-hospital cardiac arrest. Open Heart 2014;: : e000138. doi:10.1136/openhrt-2014000138

Received 24 April 2014 Revised 25 June 2014 Accepted 15 July 2014

CrossMark

For numbered affiliations see end of article.

Correspondence to Dr Thomas Werner Lindner, lindnerthomas@hotmail.com

\section{ABSTRACT}

Objective: In the last few years the genetic influence on health and disease outcome has become more apparent. The ACE genotype appears to play a significant role in the pathophysiology of several disease processes. This pilot study aims at showing the feasibility to examine the genetic influence of the ACE genotype on return of spontaneous circulation (ROSC) in out-of-hospital cardiac arrest (OHCA). Methods: We performed a prospective observational study of all OHCAs of presumed cardiac origin in a well-defined population. We collected prehospital blood samples for the determination of ACE genotype and used this information together with Utstein template parameters in a multivariable analysis to examine the relationship between ROSC and ACE genotype.

Results: We collect blood samples in 156 of 361 patients with OHCA of presumed cardiac origin, 127 samples were analysed (mean age 67 years, $86 \%$ male, $79 \%$ witnessed OHCA, $80 \%$ bystander CPR, $62 \%$ had a shockable rhythm, ROSC $77 \%$ ). Distribution of the ACE gene polymorphisms: insertion polymorphism (II) $\mathrm{n}=22,17 \%$, insertion/deletion polymorphism (ID) $\mathrm{n}=66,52 \%$ and deletion polymorphism (DD) $\mathrm{n}=39$, $31 \%$. We found no significant association between ACE II vs ACE DD/DI and ROSC (OR 1.72; Cl 0.52 to 5.73; $\mathrm{p}=0.38$ ). Other ACE genotype groupings (II/ID vs DD or II vs DD) did not change the overall finding of lack of impact of ACE genotype on ROSC.

Conclusions: This pilot study did not indicate a significant association between ACE gene polymorphism and ROSC. However, it has demonstrated that prehospital genetic studies including blood sampling are feasible and ethically acceptable.

\section{INTRODUCTION}

Despite more than 1000 cases of out-of-hospital sudden cardiac arrest (OHCA) occurring throughout Europe every day, neurologically intact survival remains poor; currently no more than $10-25 \%$ in the best emergency medical systems (EMS). ${ }^{12}$ Even when the entire chain of survival is working efficiently and effectively,

\section{KEY MESSAGES}

What is already known about this subject?

- We know the significant effects of angiotensin converting enzyme (ACE) genotype on survival from critical illness and major trauma. The ACE genotype is contributing to vascular tone and therefore we decided to perform a pilot study of the relationship between ACE genotype and return of spontaneous circulation in patients with out of hospital cardiac arrest.

What does this study add?

- This pilot study has demonstrated the feasibility of collecting blood samples from out of hospital cardiac arrest patients even if not admitted to the receiving hospital later and showed how practical, legal and ethical challenges might be overcome in such pre-hospital studies.

How might this impact on clinical practice?

- More knowledge on the genetic influence on return of spontaneous circulation rate may affect how we treat such out-of-hospital cardiac arrest patients and lead to improved prognosis.

outcome is difficult to predict, reflecting the many factors, both known and unknown, that contribute to overall survival. While many important predictors for survival are understood, such as an initial shockable rhythm, a witnessed arrest and bystander cardiopulmonary resuscitation (CPR), many other more subtle predictors are likely to be in play. ${ }^{3}$

In many areas of medicine, the genetic influence on health, disease progression and response to therapy is becoming apparent. The understanding of genetic-based alterations and diseases of the human body has expanded enormously during the past 20 years. ${ }^{4}$ Several correlations between genetic polymorphism and increased risk for disease development and outcome have 
since been established and it is likely that a genetic component determines response to some of the many pathophysiological pathways at play during attempted resuscitation from OHCA. ${ }^{5}$

An important determinant of return of spontaneous circulation (ROSC) during resuscitation from OHCA is the diastolic blood pressure generated during external cardiac chest compressions; this determining the subsequent coronary perfusion pressure. ${ }^{6}$

Vascular tone is a major factor determining diastolic blood pressure during CPR and is known to be influenced by genetic polymorphism of the angiotensin converting enzyme (ACE) gene. ${ }^{6-8}$ The peptide hormone angiotensin II (AGII) is part of the renin-angiotensinaldosterone system which controls blood pressure regulation. AGII acts to constrict vascular smooth muscle resulting in increased vascular tone and therefore increased blood pressure. The ACE gene has three genotypes: insertion polymorphism (II); insertion/deletion polymorphism (ID) and deletion polymorphism (DD). The absence (deletion, D) rather than the presence (insertion, I) of a 287 base pair fragment in the ACE gene is associated with higher circulating and tissue ACE activity. ${ }^{7}$ This genetic effect accounts for $47 \%$ of the total variance of serum ACE. ${ }^{7}$

The ACE genotype appears to play a significant role in the pathophysiology of several disease processes, although the exact mechanisms are poorly understood. In patients with the DD/DI genotype, excess mortality has been shown in adult acute respiratory distress syndrome ${ }^{9}$ and paediatric meningococcal infection ${ }^{10}$ and the genotype has also been linked to trauma survival and worse functional outcome from traumatic brain injury. ${ }^{11} 12$

In view of the significant effects on ACE genotype on survival from critical illness and major trauma and the role of the ACE genotype in determining vascular tone, we decided to perform a pilot study of the relationship between ACE genotype and outcome from OHCA of presumed cardiac cause. We felt such a pilot study focusing on feasibility could generate valuable information for future genetic OHCA studies independent of its findings. Hence, we established a study protocol with ethical approval, which made it possible to collect blood samples from OHCA victims with ongoing resuscitation or return of spontaneous circulation, to analyse the ACE genotype in OHCA victims in Western Norway.

\section{METHODS}

\section{Study design}

We performed a prospective observational study of all patients suffering OHCA in the Stavanger region of Norway; an area with a primarily Caucasian population of about $320000 .{ }^{13}$ During the study period (February 2007-November 2010), the EMS responded to 787 emergency calls to patients with OHCA, of which a

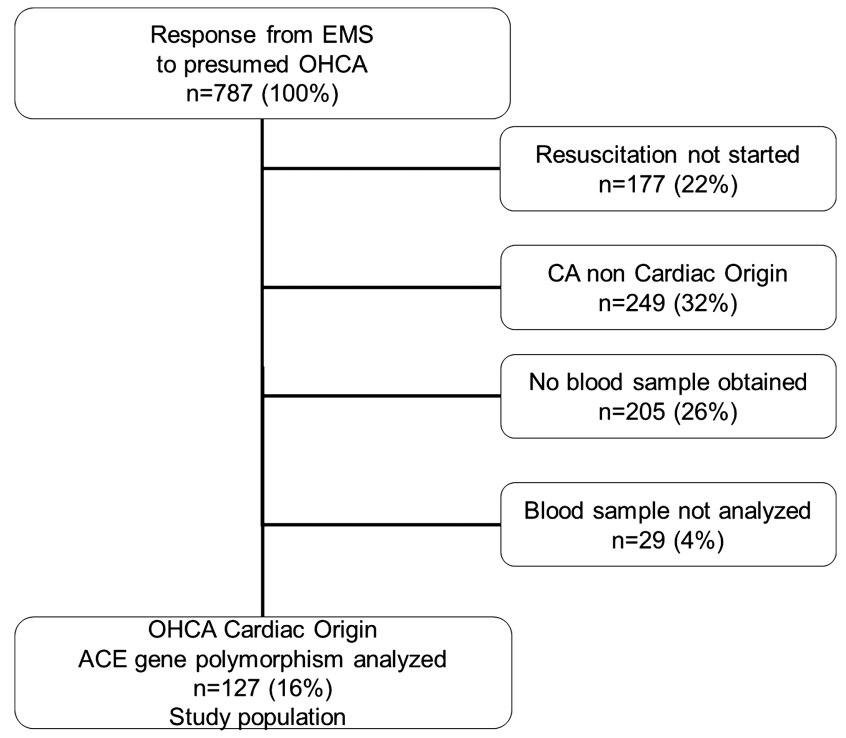

Figure 1 Flow chart of 787 patients with out-of-hospital cardiac arrest between February 2007 and November 2010

presumed cardiac origin, according to the Utstein definitions, ${ }^{14} 15$ was found in 361 patients (figure 1).

\section{System overview}

All OHCA patients with ROSC in the study region are transported to one hospital-the Stavanger University Hospital. The dispatch and EMS from the study area has been described previously. ${ }^{2}{ }^{16}$ In summary, a single alarm and dispatch centre is responsible for all call-outs of ambulance units, including a physician-manned unit, either by air or road. The alarm and dispatch centre also advises bystanders in CPR, including mouth-tomouth ventilation. System outcome data has recently been published. ${ }^{2}$

\section{Clinical care}

During the study period, all patients suffering OHCA and recruited to the study received advanced life support from the ambulance crew. In addition to the paramedic crew, a doctor was dispatched to all cases of OHCA. The 2005 European Resuscitation Council (ERC) resuscitation guidelines with Norwegian alterations were in use for all patients in this study. ${ }^{17}$

Termination of CPR mostly occurred on scene and was decided by an attending medical professional (general practitioner on call or physician-manned rapid response unit) according to local protocol. ${ }^{2}$ A minority of patients were admitted to hospital with ongoing CPR and subsequently entered into the appropriate group, depending on whether ROSC was achieved in the emergency department.

\section{Ethical considerations}

All EMS paramedics who participated in the blood sample collection process received teaching in technical and ethical aspects of the study. The study and the use 
of local databases were approved by the Norwegian Social Science Data Service, the Regional Committee for Research Ethics and the local EMS. The Regional Committee for Research Ethics accepted that no informed consent could be obtained before blood sampling from the patient or from the next-of-kin. Therefore, informed consent was obtained retrospectively, either from the patients directly if they regained the ability to consent after the treatment for their OHCA or from the next-of-kin in cases where the patient did not survive or did not make sufficient neurological recovery to give informed consent. If informed consent from next-of-kin was necessary this was obtained several weeks after the OHCA event to allow next-of-kin to recover from their severe loss. Oral and written information was given by the investigators to those giving consent and signed approval was obtained.

\section{Cardiac arrest registry}

All patients treated for OHCA by the EMS routinely have their details entered into the hospital-owned Utstein-based database. For this study the Utsteinbased $^{1415}$ database was accessed for all included patients to extract the OHCA data for further analysis. Origin of the OHCA was determined prehospital following the Utstein recommendations. ${ }^{14}{ }^{15}$ For quality assurance, data were crossed-checked before being entered in the study database. Missing data for each performed analysis are noted in the Results section

\section{Collection of genetic material}

The collection of blood samples was performed by the EMS paramedics without either disturbing or delaying the standard CPR protocol. Samples were either drawn on scene or in patients with ROSC, following transport to the hospital. Most of the blood samples were therefore obtained either after establishing ROSC or once CPR efforts were terminated. If the resuscitation efforts were terminated on scene, the deceased was, according to local law, no longer in the sphere of responsibility of the EMS. Therefore, no blood samples could be collected after death was declared by the on-scene physician. In order to improve recruitment, 10 additional blood samples were collected in the Bergen region, which have a similar EMS physician-manned service. ${ }^{18} 19$

Blood was drawn from an intravenous cannula in a peripheral vein, either from a cannula prior to administration of drugs or fluids, or from a separate unused intravenous cannula. Up to $20 \mathrm{~mL}$ venous blood was withdrawn into two $10 \mathrm{~mL}$ glass tubes coated with EDTA. These samples were transferred to the hospital, either together with the patient or by the EMS alone and, without further processing, stored at $-80^{\circ} \mathrm{C}$. The genetic analysis was performed as a single batch after all samples had been obtained.

\section{Genetic analysis}

Genomic DNA from $1 \mathrm{~mL}$ of whole blood was extracted using Qiagen Genomic-tip 20/G (QIAGEN AB; SE-19124 Sollentuna) according to manufacturer's instructions. ${ }^{20}$ The eluted DNA was precipitated and dissolved. The ACE polymorphism analysis was determined using PCR under standard conditions described elsewhere. ${ }^{21}$ One microliter of the PCR reaction product is analysed by capillary electrophoresis using a microfluidics-based platform for sizing, quantification and quality control of DNA (Agilent 2100 Bioanalyzer) to separate the $191 \mathrm{bp}$-long $\mathrm{D}$ variant from the 478 bp-long I variant.

\section{Statistical analysis}

Data were entered into a FilemakerPro7 database (FileMaker, Inc; USA), and SPSS V.20.0 (SPSS, Inc; USA) was used for statistical analysis. Figures were constructed using MicrosoftOffice 2010 (Microsoft Corporation, USA). A $\chi^{2}$ test was used to examine differences in proportions for categorical variables. Student $\mathrm{t}$ test and analysis of variance were used to examine between-group differences for continuous variables. A multiple logistic regression model was constructed. Goodness of fit for the logistic regression model was verified by the Hosmer-Lemeshow test.

Two-sided $\mathrm{p}$ values $<0.05$ were considered statistically significant. Missing data occurred in several variables examined in this study. Information on the missing data is shown in the tables if the missing data comprised more than $5 \%$ of all data collected for the variable. The numbers of complete data sets are reported for the multivariable analyses.

The presentation of the three ACE gene polymorphisms were carried out separately, but also aggregated in two groups; ACE II against ACE DD/ID as described by Kehoe. $^{12}$

\section{RESULTS}

Of the 361 patients with an OHCA of presumed cardiac origin, 156 had blood samples drawn (figure 1). A total of 127 blood samples were eligible for genetic polymorphism analysis, while a further 29 blood samples were not analysed due to lack of informed consent.

Table 1 shows the demographic and clinical characteristics of the study population and the univariate analysis of factors linked to ROSC, which was achieved in 59\% of the studied patients.

Table 2 shows the demographic and clinical characteristics of the study population stratified by the ACE genotype groups II against ID+DD, while table 3 presents the association between ACE genotype and ROSC, adjusted for demographic and clinical factors. The univariate analysis indicated a possible association between ROSC rate and ACE gene polymorphism (table 2; $\mathrm{p}=0.15$ ). However, we did not find a significant relationship between ACE genotype and ROSC in the multivariable 
Table 1 Demographic characteristics of the study population for the 'ROSC' and 'No ROSC' groups

\begin{tabular}{|c|c|c|c|c|}
\hline & $\begin{array}{l}\text { All patients } \\
n=127\end{array}$ & $\begin{array}{l}\text { ROSC } \\
n=75\end{array}$ & $\begin{array}{l}\text { No ROSC } \\
n=52\end{array}$ & p Value \\
\hline Age, year, mean (SD) & $67(15)$ & $65(14)$ & $70(15)$ & 0.042 \\
\hline Male sex, $\mathrm{n}(\%)$ & $109(86)$ & $66(88)$ & $43(83)$ & 0.399 \\
\hline Witnessed OHCA, n (\%) & $100(79)$ & $65(87)$ & $35(67)$ & 0.014 \\
\hline Bystander CPR, n (\%) & $102(80)$ & $63(84)$ & $39(75)$ & 0.210 \\
\hline Location OHCA home, n (\%) md=31 & $63(50)$ & $31(41)$ & $32(62)$ & 0.436 \\
\hline EMS response time in minutes; mean (SD) & $9(6)$ & $8(5)$ & $11(6)$ & 0.019 \\
\hline First rhythm shockable, n (\%) & $79(62)$ & $58(77)$ & $21(40)$ & $<0.001$ \\
\hline ACE II, n (\%) & $22(17)$ & $16(21)$ & $6(12)$ & 0.151 \\
\hline ACE ID, n (\%) & $66(52)$ & 37 (49) & $29(55)$ & 0.765 \\
\hline ACE DD, n (\%) & $39(31)$ & $22(29)$ & $17(33)$ & 0.162 \\
\hline
\end{tabular}

Table 2 Demographic characteristics of the study population according to the ACE genotype groups II against ID+DD

\begin{tabular}{|c|c|c|c|c|}
\hline & $\begin{array}{l}\text { All patients } \\
\mathrm{n}=127\end{array}$ & $\begin{array}{l}\text { ACE II } \\
n=22,(17 \%)\end{array}$ & $\begin{array}{l}\text { ACE ID/DD } \\
\mathrm{n}=105,(83 \%)\end{array}$ & p Value \\
\hline Age, year, mean (SD) & $67(15)$ & $69(18)$ & $67(14)$ & 0.527 \\
\hline Male sex, $n(\%)$ & $109(86)$ & $21(95)$ & $88(84)$ & 0.154 \\
\hline Witnessed OHCA, n (\%) & $100(79)$ & $17(77)$ & $83(79)$ & 0.853 \\
\hline Bystander CPR, n (\%) & $102(80)$ & $19(86)$ & $83(79)$ & 0.432 \\
\hline Location OHCA home, n (\%) md=31 & $63(50)$ & $11(50)$ & $52(50)$ & 0.946 \\
\hline EMS response time in minutes; Mean (SD) & $9(6)$ & $7(5)$ & $10(6)$ & 0.092 \\
\hline First rhythm shockable, $n(\%)$ & $79(62)$ & $15(68)$ & $64(61)$ & 0.525 \\
\hline ROSC n (\%) & $75(59)$ & $16(73)$ & $59(56)$ & 0.151 \\
\hline
\end{tabular}

Table 3 Factors associated with ROSC in the study population $(n=127)$

\begin{tabular}{llll}
\hline & OR & 95\% Cl & p Value \\
\hline Age (one additional year) & 0.98 & 0.95 to 1.00 & 0.117 \\
Gender (female) & 1.98 & 0.56 to 6.96 & 0.287 \\
Witnessed OHCA & 3.07 & 0.98 to 9.65 & 0.055 \\
Shockable rhythm & 5.28 & 2.03 to 13.72 & 0.001 \\
EMS response time (one additional minute) & 0.90 & 0.83 to 0.98 & 0.015 \\
ACE genotype & & & 0.52 to 5.73 \\
ACE II/ACE (DD+ID) & 1.72 & & 0.377 \\
\hline
\end{tabular}

Missing data 7.

ACE, angiotensin converting enzyme; DD/ID, deletion polymorphism/insertion/deletion polymorphism; II, insertion polymorphism; OHCA, outof-hospital cardiac arrest; ROSC, return of spontaneous circulation.

analysis (table 3). Comparing other ACE genotype groupings (II/ID vs DD or II vs DD) did not change the overall finding of lack of impact of ACE genotype on ROSC.

\section{DISCUSSION}

The aim of our pilot study was to show the feasibility to examine the genetic influence between ACE genotype and ROSC in OHCA of presumed cardiac origin. We could not demonstrate such a relationship between ACE genotype and ROSC in OHCA victims. Had the association of ACE genotype with ROSC been as strong as that reported with survival from major trauma, ${ }^{12}$ we would have expected to see a significant association despite the relatively small number of patients studied. Although probably related to mechanisms other than vascular tone, ACE gene polymorphism has also shown a strong association in other diseases where it determines variations in acute lung injury in sepsis, ${ }^{22} 23$ impaired lung function ${ }^{9}$ and recovery from traumatic brain injury. ${ }^{11}$ In western Caucasian population such as ours, the distribution of the ACE genotype is expected to be II $25 \%$, ID $50 \%$ and DD $25 \% .^{24-27}$ This is similar to the 
distribution found in our small patient cohort and that of the population of trauma patients demonstrating a strong association of ACE genotype with outcome. ${ }^{12}$

Recent ACE genotype research has failed to demonstrate an association with outcome in several cardiac diseases $^{28}$ and has also demonstrated the multifactorial nature of the control of vascular tone. ${ }^{29-31}$

ACE gene polymorphism controlling angiotensin pathways may only be one of several physiological mechanisms to influence the effect of vascular tone on promoting adequate diastolic blood pressure to achieve ROSC during OHCA. ACE genotype alone as a marker of risk in disease processes may well be too simplistic an approach to understanding the effects of genotype on disease processes. A more targeted approach and larger sample sizes may be appropriate for future studies. Since the inception of this study, more specific methods of analysing ACE genotype and associated metabolic pathways have become available, such as measuredhaplotype analysis of single nucleotide polymorphisms ${ }^{23}$ and future studies may be better directed using these methods.

Where genotype is associated with a specific phenotypic defect, rather than a subtle influence on metabolic pathways, a clearer association with genotype is often apparent. A number of studies have demonstrated that sudden cardiac death has a strong genetic component, particularly in association with structural defects and sudden malignant arrhythmias. ${ }^{32}$ A number of other genetic polymorphisms have all been associated with an altered risk of coronary artery disease and myocardial infarction which are indirect risk factors for cardiac arrest. ${ }^{33}$ An understanding of the genetic contributions to outcome from resuscitation could add substantially to the prediction and management of cardiac arrest and is a goal worth pursuing. We therefore believe that the main value of our pilot study is to serve as a feasibility model for future prehospital genetic studies in OHCA victims unable to give informed consent.

With regard to our study design, we believe that the present study has demonstrated the practical and ethical feasibility of collecting blood samples from patients in the prehospital arena, even if not admitted to the receiving hospital later. This design facilitates the collection of important information from OHCA victims who were declared dead on the scene even if no blood was drawn after declaration of death as described by Johnson. ${ }^{34}$ The approach used in this study can be used as an example for other studies where human material needs to be collected. The ethical details are applicable to other research studies in the prehospital arena. The ethical aspects of this study were universally accepted by EMS personal, but we found that about $20 \%$ of patients and/or relatives later declined to give informed consent. The main reason to decline informed consent from relatives was the wish not to be reminded about the death of the deceased and therefore the unwillingness of involvement. Some patients and/or relatives were uncontactable, thus preventing the use of these samples and data in the study.

In summary this demonstrates that it is possible to conduct genetic research in the ethically challenging prehospital environment with patients unable to give consent, but that studies must take into account a relatively large drop-out rate. Our experience also indicates that there may be a need for extra staff on scene, responsible solely for collection of biological specimens.

\section{Limitations}

The major limitation of this study is the small sample size, which is of particular relevance for genetic studies that often require sample size orders of a magnitude much greater than was possible in our study. ${ }^{33}{ }^{34}$ The small patient cohort studied may also explain why other factors like age and witnessed OHCA did not show significant associations with ROSC.

A main concern in the present pilot study was that the delivery of advanced life support by EMS crews should not be interrupted by the need to obtain a blood sample for the study. It was only possible to collect blood samples when enough resources were present at the OHCA scene. Several patients could not be included due to this limitation. Other ethical or logistical considerations on scene may also have influenced the inclusion of patients when effort was focussed on the resuscitation attempt. This may have inflated the proportion of patients with ROSC in our study cohort and thus limited the sample size in the comparator group.

\section{CONCLUSIONS}

This pilot study of 127 OHCA victims did not demonstrate a clear association between ACE gene polymorphism and ROSC, and was limited in its interpretation by the relatively small sample size. However, the pilot study has demonstrated the feasibility of collecting blood samples from patients with OHCA and demonstrates how practical, legal and ethical challenges might be overcome in such pre-hospital studies. More pre-hospital genetic studies in adequately powered cohorts of OHCA victims will help move forward the frontier of genetic influence on resuscitation outcomes.

\section{Author affiliations}

${ }^{1}$ Department of Anaesthesiology and Intensive Care, Stavanger University Hospital, Stavanger, Norway

${ }^{2}$ South Central Ambulance Service NHS Foundation Trust, Otterbourne, UK ${ }^{3} \mathrm{NIHR}$ Southampton Respiratory Biomedical Research Unit, Southampton University Hospital NHS Foundation Trust, Southampton, UK

${ }^{4}$ School of Health Sciences, University of Surrey, UK

${ }^{5}$ Department of Medicine, Stavanger University Hospital, Stavanger, Norway

${ }^{6}$ Department of Surgical Sciences, Anaesthesiology and Critical Care

Medicine, Uppsala University, Uppsala, Sweden

${ }^{7}$ Department of Anaesthesia and Intensive Care, Haukeland University Hospital, Bergen, Norway

${ }^{8}$ Department of Clinical Medicine, University of Bergen, Bergen, Norway 
Acknowledgements The authors are deeply grateful to the EMS employees who made this work possible. They delivered much more than expected. In addition, the authors want to thank Astrid Våge and Wenche Mathiesen for their supportive work in the analysing process and Lars Wiklund and Cecile Martijn who made the genetic analysis possible.

Competing interests None.

Ethics approval Regional committee for medical and health research ethics Bergen Norway.

Provenance and peer review Not commissioned; externally peer reviewed.

Open Access This is an Open Access article distributed in accordance with the Creative Commons Attribution Non Commercial (CC BY-NC 4.0) license which permits others to distribute, remix, adapt, build upon this work noncommercially, and license their derivative works on different terms, provided the original work is properly cited and the use is non-commercial. See: http:// creativecommons.org/licenses/by-nc/4.0/

\section{REFERENCES}

1. Berdowski J, Berg RA, Tijssen JG, et al. Global incidences of out-of-hospital cardiac arrest and survival rates: systematic review of 67 prospective studies. Resuscitation 2010;81:1479-87.

2. Lindner TW, Soreide E, Nilsen OB, et al. Good outcome in every fourth resuscitation attempt is achievable-an Utstein template report from the Stavanger region. Resuscitation 2011;82:1508-13.

3. Sasson C, Rogers MA, Dahl J, et al. Predictors of survival from out-of-hospital cardiac arrest: a systematic review and meta-analysis. Circ Cardiovasc Qual Outcomes 2010:3:63-81.

4. Lander ES, Linton LM, Birren B, et al. Initial sequencing and analysis of the human genome. Nature 2001;409:860-921.

5. Royse SD. Implications of genetic testing for sudden cardiac death syndrome. Br J Nurs 2006;15:1104-7.

6. Paradis NA, Martin GB, Rivers EP, et al. Coronary perfusion pressure and the return of spontaneous circulation in human cardiopulmonary resuscitation. JAMA 1990;263:1106-13.

7. Rigat B, Hubert C, Alhenc-Gelas F, et al. An insertion/deletion polymorphism in the angiotensin I-converting enzyme gene accounting for half the variance of serum enzyme levels. $J$ Clin Invest 1990;86:1343-6.

8. Tiret L, Rigat B, Visvikis S, et al. Evidence, from combined segregation and linkage analysis, that a variant of the angiotensin l-converting enzyme (ACE) gene controls plasma ACE levels. Am J Hum Genet 1992;51:197-205.

9. Marshall RP, Webb S, Bellingan GJ, et al. Angiotensin converting enzyme insertion/deletion polymorphism is associated with susceptibility and outcome in acute respiratory distress syndrome. Am J Respir Crit Care Med 2002;166:646-50.

10. Harding D, Baines PB, Brull D, et al. Severity of meningococcal disease in children and the angiotensin-converting enzyme insertion/ deletion polymorphism. Am J Respir Crit Care Med 2002;165:1103-6.

11. Ariza $M$, Matarin MD, Junque $C$, et al. Influence of angiotensin-converting enzyme polymorphism on neuropsychological subacute performance in moderate and severe traumatic brain injury. J Neuropsychiatry Clin Neurosci 2006;18:39-44

12. Kehoe AD, Eleftheriou $\mathrm{KI}$, Heron $\mathrm{M}$, et al. Angiotensin-converting enzyme genotype may predict survival following major trauma. Emerg Med J 2008;25:759-61.

13. Statistics-Norway. Population Figures Statistics Norway. http://www. ssb.no/english/subjects/02/befolkning_en/ 2009 (accessed 2009).

14. Cummins RO, Chamberlain D, Hazinski MF, et al. Recommended guidelines for reviewing, reporting, and conducting research on in-hospital resuscitation: the in-hospital 'Utstein style'. American Heart Association. Circulation 1997;95:2213-39.
15. Jacobs I, Nadkarni V, Bahr J, et al. Cardiac arrest and cardiopulmonary resuscitation outcome reports: update and simplification of the Utstein templates for resuscitation registries. A statement for healthcare professionals from a task force of the international liaison committee on resuscitation (American Heart Association, European Resuscitation Council, Australian Resuscitation Council, New Zealand Resuscitation Council, Heart and Stroke Foundation of Canada, InterAmerican Heart Foundation, Resuscitation Council of Southern Africa). Resuscitation 2004;63:233-49.

16. Langhelle A, Tyvold SS, Lexow K, et al. In-hospital factors associated with improved outcome after out-of-hospital cardiac arrest. A comparison between four regions in Norway. Resuscitation 2003:56:247-63.

17. Lexow K, Sunde K. Why Norwegian 2005 guidelines differs slightly from the ERC guidelines. Resuscitation 2007;72:490-2.

18. Heradstveit BE, Sunde K, Sunde GA, et al. Factors complicating interpretation of capnography during advanced life support in cardiac arrest-a clinical retrospective study in 575 patients. Resuscitation 2012;83:813-18.

19. Lindner T, Langorgen J, Sunde $\mathrm{K}$, et al. Factors predicting the use of therapeutic hypothermia and survival in unconscious out-of-hospital cardiac arrest patients admitted to the ICU. Critical care 2013;17: R147.

20. QIAGEN. QIAGEN Genomic DNA Handbook. Secondary QIAGEN Genomic DNA Handbook 1999-2012. http://www.qiagen.com/ knowledge-and-support/resource-center/resource-download.aspx? id=402bb209-4104-4956-a005-6226ff0b67d5\&lang=en (accessed 2013).

21. Takemoto $\mathrm{Y}$, Sakatani M, Takami S, et al. Association between angiotensin II receptor gene polymorphism and serum angiotensin converting enzyme (SACE) activity in patients with sarcoidosis. Thorax 1998:53:459-62.

22. Jerng JS, Yu CJ, Wang HC, et al. Polymorphism of the angiotensin-converting enzyme gene affects the outcome of acute respiratory distress syndrome. Crit Care Med 2006;34:1001-6.

23. Nakada TA, Russell JA, Boyd JH, et al. Association of angiotensin II type 1 receptor-associated protein gene polymorphism with increased mortality in septic shock. Crit Care Med 2011;39:1641-8.

24. Cambien F, Poirier O, Lecerf L, et al. Deletion polymorphism in the gene for angiotensin-converting enzyme is a potent risk factor for myocardial infarction. Nature 1992;359:641-4.

25. Danser AJ, Schalekamp MA, Bax WA, et al. Angiotensin-converting enzyme in the human heart effect of the deletion/insertion polymorphism. Circulation 1995;92:1387-88.

26. Marian $\mathrm{A}, \mathrm{Yu} \mathrm{Q}$, Workman $\mathrm{R}$, et al. Angiotensin-converting enzyme polymorphism in hypertrophic cardiomyopathy and sudden cardiac death. Lancet 1993;342:1085-86.

27. Schunkert H, Hense H-W, Holmer SR, et al. Association between a deletion polymorphism of the angiotensin-converting-enzyme gene and left ventricular hypertrophy. New Engl J Med 1994:330:1634-38.

28. Bernstein KE, Ong FS, Blackwell WL, et al. A modern understanding of the traditional and nontraditional biological functions of angiotensin-converting enzyme. Pharmacol Rev 2013:65:1-46

29. Rieder MJ, Taylor SL, Clark AG, et al. Sequence variation in the human angiotensin converting enzyme. Nat Genet 1999;22:59-62.

30. Sayed-Tabatabaei FA, Oostra BA, Isaacs A, et al. ACE polymorphisms. Circ Res 2006;98:1123-33.

31. Wu CK, Tsai CT, Chang YC, et al. Genetic polymorphisms of the angiotensin II type 1 receptor gene and diastolic heart failure. $J$ Hypertens 2009;27:502-7.

32. Noseworthy PA, Newton-Cheh C. Genetic determinants of sudden cardiac death. Circulation 2008;118:1854-63.

33. Yende S, Wunderink RG. Genetic polymorphisms that predict outcome and need for treatment in cardiovascular disease. Curr Opin Crit Care 2006;12:420-5.

34. Johnson $\mathrm{C}$, Lemaitre $\mathrm{R}$, Fahrenbruch $\mathrm{C}$, et al. Common variation in fatty acid genes and resuscitation from sudden cardiac arrest. Circ Cardiovasc Genet 2012;5:422-9. 